\title{
Aspekte der Brachlandentwicklung im Schweizer Alpenraum 1950-1980
}

\section{Problemstellung und Zielsetzung}

Unter dem Begriff «Brachland» versteht man heute mehrheitlich «nicht mehr genutztes landwirtschaftliches Kulturland». In der Schweiz wurde auf die aus Brachland erwachsenden Probleme erstmals von SURBER et. al. (1973) hingewiesen. Einige Gedanken zum Fragenkreis "Problematisierung von Brachland» erscheinen zum Beginn dieses Themenheftes sinnvoll, da heute in diesem Punkt weitgehende Uneinigkeit herrscht. Es läßt sich beobachten, daß je nach Berufsgruppe (v.a. Land- und Forstwirtschaft) und auch nach persönlichem Erfahrungsbereich (regional differenziert) die Brachlandentwicklung sowohl überproblematisiert als auch banalisiert wird. Aus geographischer Sicht stellt sich also das Problem, daß analytisch erarbeitete Resultate zur Beurteilung der Brachlandentwicklung, die eine differenzierte Beurteilung über größere Räume erlauben, heute weitgehend fehlen.

Zielsetzung dieser Arbeit ist es, einerseits Ansätze zu einer Theorie der Brachlandentwicklung aufzuzeigen, was dazu dienen soll, aktuelle und zukünftige Problemgebiete zu definieren (Kap.2). Andererseits sollen Wege zu einer Beurteilung der räumlichen Entwicklungen aufgezeigt (Kap.3) und erste vergleichende Bewertungen formuliert werden (Kap.4). Die relativ theoretischen Gedanken sollen dem Praktiker und Politiker räumliche Entwicklungen aufzeigen, die im Rahmen der Nutzungs- und Richtplanung behandelt werden sollten. Die im Text formulierten Thesen beziehen sich auf umfangreiche Studien, die in zahlreichen Untersuchungsgebieten (Fig. 1) im Schweizer Alpenraum durchgeführt wurden (vgl. auch Literaturverzeichnis). In diesen Untersuchungsgemeinden wurde die Brachlandentwicklung 1950-1980 nach einer in WALTHER/JULEN (1983) beschriebenen Methodik analysiert und bewertet. Daß die formulierten Gedanken auch eine gewisse Praxistauglichkeit aufweisen, haben wir in einem weiteren Artikel aufzuzeigen versucht, wo eine praktische Methode zur Erfassung und Beurteilung von Brachlandsituationen im Rahmen der Richt- und Nutzungsplanung vorgestellt wird (HUGENTOBLER/WALTHER 1983).

\section{Brachlandentwicklung und sozialer Wandel in der Landwirtschaft}

\subsection{Eine Brachland-Entwicklungs-Theorie}

Brachland ist eine Erscheinungsform raumwirksamer Prozesse, deren Ursachen vor allem im umfassenden sozialen Wandel innerhalb der Landwirtschaft $\mathrm{zu}$ suchen sind. Erklärungsansätze finden wir einerseits im Wertvorstellungswandel selbst (Technisierung, Wandel in der Produktionsstruktur usw.), andererseits zu einem überwiegenden Teil in Anpassungsproblemen an diesen gesamtgesellschaftlichen Wandel. Daraus wird klar, daß die Brachlandentwicklung nicht isoliert, sondern im weiteren Zusammenhang der Suche nach einer standortgerechten Nutzung (Intensivierung versus Extensivierung) zu betrachten ist.

Die wichtigsten Elemente des sozialen Wandels auf Ebene der Landwirtschaftsbetriebe lassen sich wie folgt zusammenfassen: Im Laufe der letzten Jahrzehnte mußte ein großer Teil der Arbeitskräfte durch Maschinen ersetzt werden (EVD 1976), was unter anderem auch einen Zwang zur Spezialisierung der Produktionsrichtung und zur Betriebsvergrößerung zur Folge hatte. Der Wandel von einer traditionsgeleiteten in eine technologisch adaptierte Agrargesellschaft wurde auch durch die enorme agrartechnologische Entwicklung (futterbauliche Verbesserungen, Bewirtschaftungstechnologien usw.) unterstützt. Der Bauer wurde zum Unternehmer, der nach ökonomischen Kriterien Ertrag und Grenzertrag kalkuliert.

Wo die Voraussetzungen für diese den Leitbildern entsprechenden Modernisierungsprozesse gegeben sind, entwickelte sich die Flurbewirtschaftung zu einer standortgerechten Nutzung. Zentrale Voraussetzungen sind vor allem die Anpassungsmöglichkeit der landwirtschaftlichen Betriebsstruktur (Produktionsrichtung, Kapital, Gebäudeinfrastruktur usw.) und im sozialen Bereich die Adaption der Grundbesitzstruktur und die Innovationsfreude. Mechanisch nicht bewirtschaftbare Parzellen werden beweidet, und die Wirtschaftsfläche wird im Zuge der Rationalisierung zentriert. Eine Betriebsaufgabe hat zur Folge, daß die

Pierre Walther, Stefan Julen,

Geographisches Institut der Universität Zürich-Irchel, Winterthurerstr. 190, 8057 Zürich 
verbleibenden Betriebe durch Zupacht ihre Wirtschaftsfläche aufstocken können. In diesen Gebieten findet sich Brachland nur vereinzelt auf nicht beweidbaren Standorten, Extremflächen, sowie auf kleinen, unerschlossenen Schnittwiesen in der Maiensäß- und Bergwiesenstufe. Diese Brachlandsituation ist typisch für viele Regionen im Schweizer Berggebiet und läßt sich in Fig. 2 etwa für die Gemeinden Riemenstalden, Camuns, Castiel und Ramosch bestätigen.

Wo die sozialen und ökonomischen Voraussetzungen für diese Modernisierung nicht gegeben sind, folgt die Brachlandentwicklung anderen Gesetzmäßigkeiten. Eine Betriebsaufgabe führt zu einer Auflassung der Nutzparzellen, die anschließend wegen fehlender Pachtland-Nachfrage oder wegen eines ungünstigen sozialen Klimas ungenutzt bleiben. Resultat ist eine parzellenartige Brachlandverteilung als Spiegel der Sozialstruktur der Grundbesitzer, die sich im Laufe der Zeit zu einer flurartigen verdichtet (vgl. Kap. 2.2). In kurzer Zeit werden auch beste landwirtschaftliche Böden aufgegeben (vgl. Fig. 2: Vernamiège, Chandolin und Palgnedra).
Aus diesen Ausführungen wird klar, daß eine Brachland-Entwicklungs-Theorie zentral mit der Adaptionsfähigkeit eines landwirtschaftlichen Systems an die wechselnde gesellschaftliche Umwelt zu verbinden ist. Anpassungsprobleme liegen sowohl im inneren System des Landwirtschaftsbetriebes (Infrastruktur, Produktionsstruktur) wie vor allem auch im sozialen Bereich (kollektive Wertvorstellungen, Besitzstruktur usw.).

\subsection{Realteilungsgebiete als Problemgebiete}

Eine Folgerung aus der skizzierten Entwicklungstheorie ist klar: Größte Brachland-Problemgebiete sind die Realteilungsgebiete (große Teile der Kantone Graubünden, Wallis und Tessin), weil hier die Anpassungsprobleme in verschiedenen Formen auftreten (vgl. Fig. 2). Der Begriff Realteilung bezieht sich vorerst einmal auf das bäuerliche Erbrecht mit seinen direkten Folgen der Besitzzersplitterung in Flur- und Gebäudeanteile. Wichtig sind aber vor allem die daraus erwachsenden Folgeprobleme wie kleine,

Fig. 1 Untersuchungsgebiete im Schweizer Alpenraum

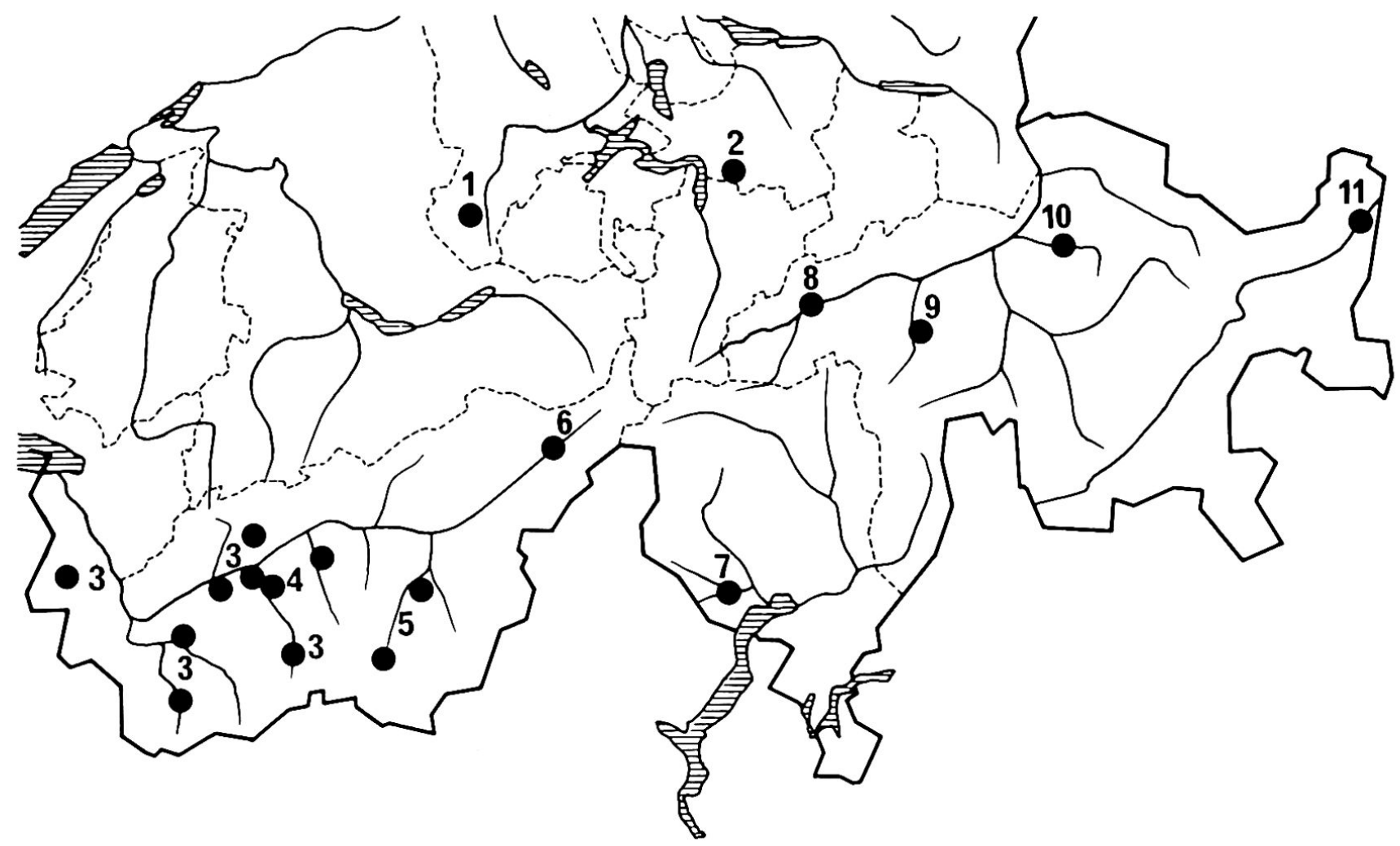

Legende:

1 Flühli/Sörenberg

2 Riemenstalden

3 Mittel- und Unterwallis: Champéry, Bourg St. Pierre, Verbier, Nendaz, Vex, Ayent, Arolla, Chandolin

4 Val d'Hérens: Nax, Vernamiège, Mase, St. Martin (Hirzel 1983)

5 Mattertal: Grächen, Randa, Täsch, Zermatt
6 Obergoms: Oberwald - Niederwald (Walther/Gresch/ Haefner 1982)

7 Centovalli: Borgnone, Intragna, Palagnedra (Mahler 1981)

8 Vorderrheintal: Schlans, Truns, Somvix

9 Lugnez: Duvin, Camuns, Tersnaus, St. Martin (Räber 1981)

10 Schanfigg: Maladers, Calfreisen, Castiel, Lüen, Pagig, St. Peter (Ruf 1982)

11 Unterengadin: Sent, Ramosch, Tschlin (Schori 1982) 


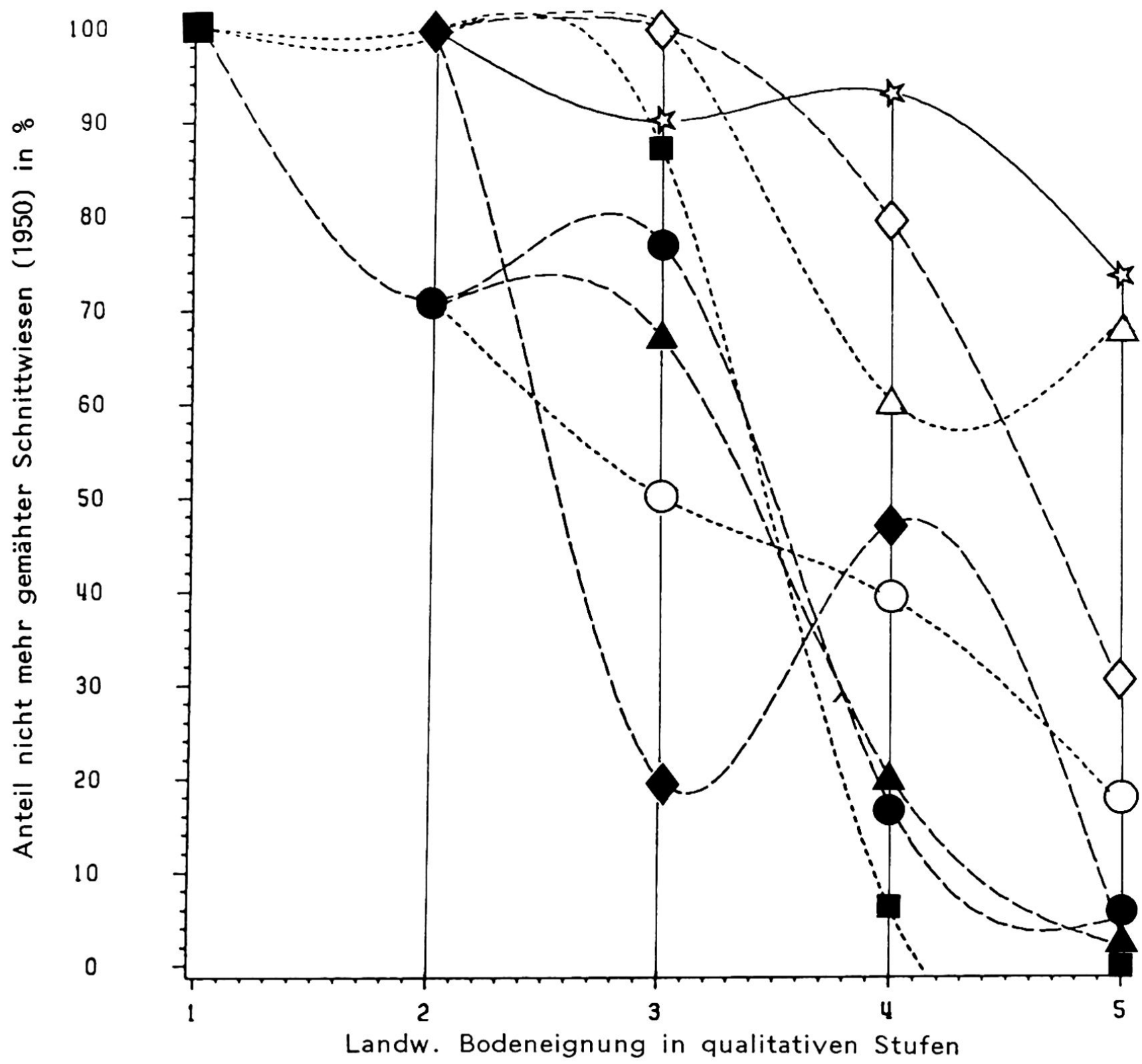

1 Wildheuflächen, Bergwiesen (nicht mechanisierbar)

2 Maiensäße (nicht mechanisierbar)

3 Talstufe (nicht mechanisierbar)

4 Maiensäße (mechanisierbar)

5 Talstufe (mechanisierbar)

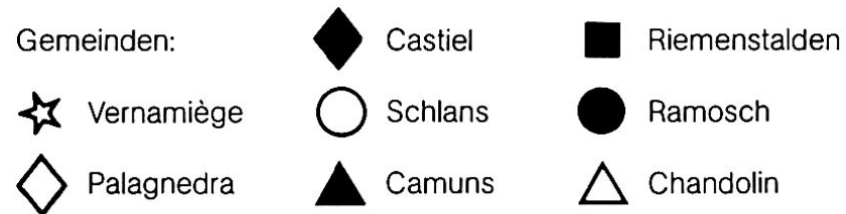

kapitalarme Betriebe, diversifizierte Produktionsstruktur, arbeitsintensive Besitzverteilung in verschiedenen Höhenstufen und Durchmischung der Wertvorstellungen.

Entscheidend für die Überwindung dieser Probleme sind nun einerseits ihr reales Ausmaß und andererseits die spezifischen, regional verschiedenen Voraussetzungen für deren Überwindung. Zentrale Voraussetzungen sind das Kapital und die kollektive Wertvor- stellung mit dem Ziel, eine lebensfähige Landwirtschaft zu erhalten. Es wird klar, daß mit diesen Überlegungen die Verbindung zu gesellschaftswissenschaftlichen Fragestellungen gegeben ist: Fragen der Zentrum-Peripherie-Problematik, der regionalen Identität, der sozialen Schichtung sowie spezifisch agrarsoziologische Probleme haben in der Erklärung der Brachlandentwicklung einen zentralen Stellenwert. Gerade typisch ist für Realteilungsgebiete die Durch- 
mischung von verschiedenen Wertvorstellungen, was in vielen Fällen einer Brachlandentwicklung förderlich ist. Diese Problematik ist noch verstärkt in Gebieten mit außerlandwirtschaftlichen, spekulativen Interessen am Grundbesitz (v. a. Tourismusgebiete).

Auf Grund des Gesagten ist eine klare Differenzierung möglich in Realteilungsgebiete, die auf sozialer Ebene Innovationen, wie Verpachtung, Pachtlandarrondierungen usw., durchführen konnten, und in solche mit schwerwiegenderen Adaptionsproblemen. Erstere sind überwiegend Gebiete mit einer gesunden Betriebsstruktur. In diesen Gebieten folgt die Brachlandentwicklung den oben beschriebenen GesetzmäBigkeiten, d. h. die nicht maschinell bewirtschaftbaren und die peripheren Wirtschaftsflächen werden als erste aufgelassen (Fig. 2). Sobald Brachland auf landwirtschaftlich geeignetem Boden auftritt, werden Maßnahmen zu einer zumindest noch extensiven Weidenutzung ergriffen. In Gebieten, wo diese Voraussetzungen nicht gegeben sind (v.a. Wallis und Tessin), ist eine charakteristische kausale Abfolge der Brachlandentwicklung beobachtbar, die zwangsläufig über ein Stadium der Aufgabe ganzer, auch bestens geeigneter Flurteile führt. Am Beispiel des Obergoms haben wir versucht, die Stadien dieser Abfolge als räumlichen Prozeß zu erfassen (WALTHER/GRESCH/ HAEFNER 1982). Es zeigte sich, daß vor allem das Beharren einzelner Landwirte auf alten, traditionsgeleiteten Bewirtschaftungsformen (z. B. Ackerbau auf Kleinstparzellen) jeden Schritt zu einer standortgerechten Flurnutzung unterbindet und somit paradoxerweise der Brachlandentwicklung entscheidenden Vorschub leistet. Erst im Stadium der totalen Fluraufgabe entsteht ein Freiraum für eine angepaßtere, leider meist nur noch extensive Flurnutzung. Leider ist in diesem Zeitpunkt die Verwaldung meist schon weit fortgeschritten, womit klar gesagt ist, daß der Handlungsspielraum für planerische und kulturtechnische Eingriffe in solchen Problemflurteilen durch die naturgegebene Dynamik des Holzgewächs-Aufwuchses begrenzt wird.

\subsection{Entwicklungstendenzen}

In den bisherigen Ausführungen wurde der soziale Wandel, wie er auch von staatlicher Seite unterstützt wird, als Erklärungsgrundlage verwendet. Es stellen sich nun die Fragen, ob regional verschieden mit gewissen lags (Verzögerungen) in der Brachlandentwicklung zu rechnen ist oder ob die Diffusion neuer Wertvorstellungen auf individueller oder staatlicher Ebene zu einer Wende in der ganzen Entwicklung führen könnte. Aus der Beantwortung dieser Fragestellungen könnten eventuell Prognosen über die künftige Brachlandentwicklung abgeleitet werden.

Wir vertreten die These, daß vor allem in heute stark überalterten Gebieten mit einer zunehmenden Brachlandentwicklung $\mathrm{zu}$ rechnen ist, da die skizzierte
Entwicklung durch die Beibehaltung von traditionellen Wertvorstellungen verzögert werden kann. Im Zuge des Generationenwechsels ist in diesen Gebieten also mit einer zunehmenden Brachlandentwicklung zu rechnen. Eine delikatere Frage ist, ob eventuell die Nebenerwerbslandwirtschaft zu einem Entwicklungs-Lag führt. Im Val d'Hérens konnte HIRZEL (1983) feststellen, daß im Zuge des Generationenwechsels die Nebenerwerbslandwirtschaft zurückging und die Brachlandentwicklung deshalb stark voranschritt. LANDTwING (1979) stellte fest, daß die Nebenerwerbslandwirte in der Industrie meist einen unqualifizierten Arbeitsplatz besetzen, die junge Generation jedoch durch ihre Ausbildung eine sozial bessere Position anstrebt und deshalb den landwirtschaftlichen Nebenerwerb aus Zeitgründen aufgibt. Eine abschließende Beurteilung dieser Probleme überfordert unsere Möglichkeiten, doch scheint uns das Problem eventueller Wertvorstellungsdifferenzen zwischen den Generationen die entscheidende Frage zu sein.

In den letzten Jahren gewann weiter die Verbreitung neuer Wertvorstellungen und Organisationsformen innerhalb der Landwirtschaft eine für die Erklärung der Brachlandentwicklung entscheidende Bedeutung. Dabei kann generell unterschieden werden zwischen einem Wertvorstellungswandel innerhalb der Bevölkerung (individuelle Ebene) und neuen Wertvorstellungen, die von amtlicher Seite vertreten werden (behördliche und staatliche Ebene). DARBELLAY (1980) vertritt die Ansicht, daß die Landwirtschaftspolitik vermehrt im Kontext der gesamtgesellschaftlichen Diskussionen (Arbeitslosigkeit, Lebensqualität usw.) diskutiert werden sollte. Der Aspekt «Landwirtschaft als sicherer Arbeitsplatz» könnte in Zukunft eine zunehmende Bedeutung erhalten. In diesem Zusammenhang könnte wiederum die Nebenerwerbslandwirtschaft und weiter die Freizeitlandwirtschaft die Brachlandentwicklung beeinflussen, vor allem da diese Bauern dem Gedanken der Landschaftspflege meist aufgeschlossen sind. Die zentrale Frage ist, als wie dauerhaft sich diese Betriebsformen erweisen, was am Beispiel der Alternativbauern, die in gewissen Regionen des Berggebietes (v. a. Tessin) seit Jahren keine vernachlässigbare Minderheit mehr sind (vgl. MAHLER 1981), gut illustriert werden kann. Auf betrieblicher Ebene ist ferner die Diffusion neuer Organisationsformen zu beachten. DARBELlaY berichtet an dieser Tagung über Erfahrungen mit Gemeinschaftsställen; ein weiterer Teilnehmer dieses Symposiums, $\mathrm{NEF}$, hat in einem touristisch entwickelten Gebiet einen Lohnarbeiterbetrieb zur Pflege des Landschaftsbildes eingerichtet. Diese innovativen Modelle könnten einen bedeutenden Einfluß auf die zukünftige Brachlandentwicklung haben. Des weiteren läßt sich ein Wertvorstellungswandel auch in der staatlichen Subventionspolitik feststellen. Der Milchwirtschaftsbeschluß (1977) und die Auszahlung der Flächenbeiträge (1979) können als raumwirksame Entscheide 
eingestuft werden, deren tatsächliche Auswirkung auf die Brachlandentwicklung noch nicht endgültig gesichert ist. Wir fragen uns, ob die Brachlandentwicklung, die doch eindeutig auf einen tiefliegenden Wertvorstellungswandel zurückzuführen ist, allein durch staatliche Maßnahmen (z.B. wirtschaftliche Anreize) gesteuert werden kann.

\section{Brachland als Umweltveränderung}

\subsection{Dimensionen von "Umweltveränderung»}

Der beschriebene soziale Wandel führt zu einer umfassenden Veränderung der Umwelt, wobei die Brachlandentwicklung nur ein Teilaspekt dieses Wandels ist. Im folgenden möchten wir vom Ansatz ausgehen, da $\beta$ Brachland als Umweltveränderung interpretierbar und beurteilbar ist. Dazu sind zuerst einmal einige Betrachtungen zum Begriff «Umweltveränderung» nötig, und anschließend muß Brachland in relevante und beurteilbare Umweltkategorien unterteilt werden (vgl. Kap. 3.2). Ziel ist eine differenzierte Betrachtung und Beurteilung der Brachlandsituationen (Kap. 4), die über eine rein flächenmäßige Erfassung der aufgelassenen Flächen (SURBER et al. 1973) hinausgehen soll.

Erstens sei gesagt, daß sich der Begriff «Umwelo» immer auf bestimmte soziale Gruppen zu beziehen hat. Ein Forstingenieur zum Beispiel wird die Veränderungen in seiner Umgebung ganz anders wahrnehmen als etwa ein Tourist. Gerade aus der Verschiedenheit von Beurteilungspositionen ergeben sich zahlreiche Konflikte und Beurteilungsdifferenzen. Zweitens ist wichtig, daß die Begriffe "Umwelt, Umweltveränderung» in ganz verschiedene Dimensionen und Aspekte aufgegliedert werden können (WEICHHART 1980), was übrigens auch in den Beiträgen dieses Themenheftes klar zum Ausdruck kommt. Umwelt kann einerseits als natürliches, ökologisches System betrachtet werden, womit Fragestellungen wie zum Beispiel «Einfluß der Brachlegung auf den Bodenwasserhaushalt» angesprochen sind. Viel wichtiger erscheint jedoch in der momentanen Diskussion der Aspekt «Umweltveränderung als Änderung der Ressourcen, Wirtschaftsflächen für die Gesellschaft» zu sein. In diesem Zusammenhang stehen die Fragen «Brachland als Waldflächenentwicklung» und "Brachland als Verlust landwirtschaftlicher Ressourcen» im Vordergrund. Ein weiterer Aspekt ist schließlich die psychische Dimension der Umwelt: Umwelt als Erlebnisbereich der Gesellschaft. Hier wiederum stehen Fragen wie «Brachland und Landschaftsbild», "Brachland als Freiraum für die Gesellschaft» im Zentrum, wobei festzuhalten ist, daß das "Landschaftsbild» gerade in touristischen Gebieten als wirtschaftliche Ressource zu betrachten ist.

$\mathrm{Zu}$ einer objektiven, die verschiedenen Umwelt-Dimensionen berücksichtigenden Aussage sind zuerst einmal die Grundbegriffe zu erarbeiten, mit denen verschiedene Brachland-Situationen miteinander verglichen werden können. Im folgenden Kapitel soll ein Ansatz zu einer solchen Begriffsbildung vorgestellt werden.

\subsection{Brachland-Kategorien}

Brachland ist keine Einheits-Kategorie, sondern kann nach verschiedenen Kriterien gegliedert werden. Im Rahmen eines Projektes haben wir im Schweizer Alpenraum ca. 400 Brachflächen inventarisiert und anschließend mit dem Ziel einer Begriffsbildung ausgewertet. Eine illustrierte Zusammenstellung der Resultate haben wir in einem Bericht (WALTHER/JULEN 1983) veröffentlicht. An dieser Stelle sollen in kurzer Form einige Grundgedanken dieser Kategorien-Bildung erläutert werden.

Wir kamen zur Überzeugung, daß die Begriffsbildung in zwei räumliche Betrachtungsebenen, in eine standörtliche (Formationenebene) und eine räumliche (Verteilungsebene), aufzutrennen ist. In der Formationenebene werden die Vegetationszustände auf Brachland nach ihrem Erscheinungsbild typisiert, in der Verteilungsebene einerseits die Verteilung Brachland landwirtschaftlich genutzte Fläche - Wald und andererseits die Verteilung von holzgewächsbestocktem zu holzgewächsfreiem Brachland untersucht. Die Trennung in diese Betrachtungsebenen erlaubt detaillierte Beurteilungen und schließlich auch planerische Aussagen.

Auf der Formationenebene wird mit Hilfe metrischer Kriterien (MAHRER 1976) und der Artenzusammensetzung die Brachlandformation beschrieben. Gras-/ Kraut-Formationen sind zum Beispiel ohne weiteres wieder in landwirtschaftliches Kulturland rückführbar, während holzgewächsbestockte Flächen, die in strauch- und baumschichtdominante Formationen aufgeteilt werden können, unter den heutigen Umständen irreversibel zu Brachland geworden sind. Der Holzgewächs-Aufwuchs läßt sich in seiner Artenzusammensetzung nach gewissen Eigenschaften gliedern: Lockerbusch- und Lockerwald-Formationen haben zum Beispiel einen gewissen ökologischen Wert, vor allem wenn sie gehölzartig aufwachsen, während Baumjungwuchs- und Jungwald-Formationen eher forstwirtschaftlich interessant sind. Völlig wertlos sind hingegen Dichtbusch- und Gebüschwald-Formationen.

Bei der Bildung der Verteilungstypen sind wir von den planerisch wichtigen Kriterien Größe, Lage zum Wald und Form der Brachlandflächen ausgegangen (BARDELEBEN/GEKLE 1978). Diese Kriterien spielen in der Beurteilung und Behandlung des Brachlandes eine große Rolle. Größere Brachlandflächen werden zum Beispiel mit den Begriffen randlagig, flur- oder komplexartig charakterisiert, kleinflächig sind parzellen-, saum-, lichtungs- und heckenartige Brachlandverteilungen. 
Fig. 3 Brachlandanteile in verschiedenen Bodeneignungsklassen

Gemeinden Landw. Bodeneignung

VERNAMIEGE

CAMUNS

CASTIEL

SCHLANS

3

VI

3

3

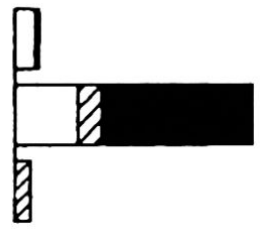

3

1

2

3
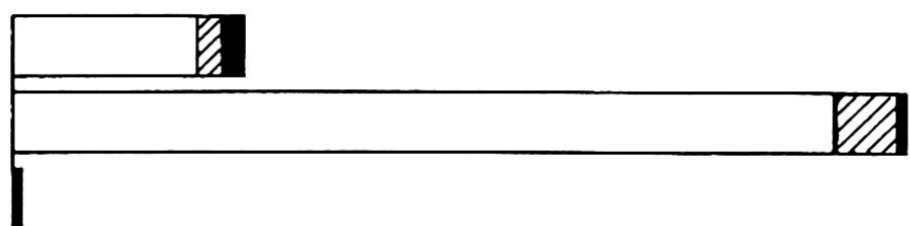

1

2

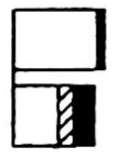

PALAGNEDRA

1

2

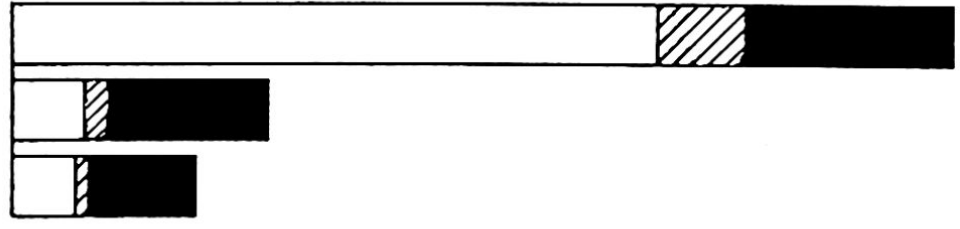

3

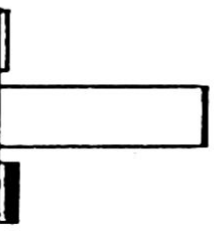

RIEMENSTALDEN

RAMOSCH

\section{CHANDOLIN}

$$
1
$$

2

3

1

2

3

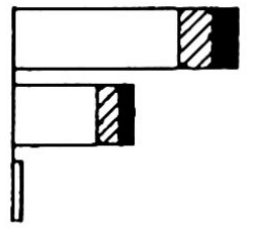

1

2

3

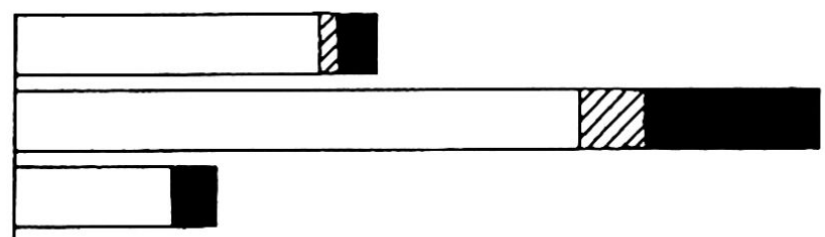

$\begin{array}{lllllllll}50 & 100 & 150 & 200 & 250 & 300 & 350 & 400 & 450\end{array}$

Promille der landwirtschaftlichen Nutzfläche 1950

Legende:

Landw. Bodeneignung:

1 mechanisierbar

2 nicht mechanisierbar

3 unerschlossen

Formationen:

$\square$ gras-/krautschicht-dominante Formationen

QZZZZ strauchschicht-dominante Formationen

baumschicht-dominante Formationen 
Richtig beurteilbar werden die Umweltveränderungen schließlich mit der Überlagerung der Brachlandsituation mit Daten zum Beispiel der landwirtschaftlichen Bodeneignung. Mit Hilfe dieser Methode werden differenzierte Fragestellungen (vgl. Fig. 3 und Kap.4) beantwortbar.

\section{Beurteilungen}

\subsection{Brachland als Veränderung im Naturraum}

$\mathrm{Zu}$ diesem Punkt sollen hier nur einige wenige Bemerkungen gemacht werden, da die Beiträge von ZOLLER und KARL direkt auf diese Thematik Bezug nehmen. Zwei Fragen stehen dabei im Vordergrund: Wie ist die Brachlandentwicklung aus Sicht des Naturschutzes zu bewerten, und wie ist die Entwicklung von allfälligen Realschäden auf Brachland zu beurteilen?

Aus der Sicht des Naturschutzes läßt sich feststellen, $\mathrm{da} \beta$ in praktisch allen untersuchten Gebieten in den ersten Stadien der Brachlandentwicklung ein ökologischer Gewinn zu beobachten ist. Vor allem in waldarmen Gebieten ist der gehölzartige Aufwuchs, vor allem von artenreichen Lockerbusch-Formationen, wertvoll (z. B. Schlans, Ramosch). Dabei sind trokkene, langsamwüchsige Gebiete gegenüber raschwüchsigen Klimaregionen (z. B. Tessin) im Vorteil, und man kann aussagen, daß der ökologische Gewinn in der kollinen und montanen Stufe größer ist als in den subalpinen Gürteln, wo praktisch ausschließlich flächenbildender Nadelholzjungwuchs aufwächst. Problematisch wird die Beurteilung in den fortgeschrittenen Stadien der Brachlandentwicklung: Die flurartige Verteilung von Lockerbusch- und Gebüschwald-Formationen, wie sie zum Beispiel im Wallis oftmals auftreten, können auch in ihrer Artenvielfalt kaum mehr als ökologischer Gewinn bezeichnet werden, da die landschaftliche Vielfalt (Verteilungsebene) verlorengeht. Die Beurteilung der Umweltveränderungen steht unserer Meinung nach also in einem direkten Zusammenhang zur kausalen Abfolge der Brachlandentwicklung (Kap. 3) und zur Verteilung der aufgelassenen Flächen im Raum.

Etwas differenzierter muß die Entwicklung der Realschadengefahr (Erosions- und Brandgefahr) betrachtet werden. Die Gefahr der Rutschungs- und Lawinenbildung wird vor allem bei einer großflächigen Verteilung von Gras-/Kraut-Formationen an steilen Hängen erhöht. Wie KARL festhält, ist jedoch die Disposition des Naturraumes (geologische Verhältnisse usw.) ein wichtiger Faktor in der Beurteilung. Unserer Meinung nach ist der Erosionsentwicklung auf steilen Bergwiesen oberhalb der Waldgrenze besondere Beachtung zu schenken, gerade weil diese Flächen schon in praktisch allen Gebieten brachgelegt sind (Fig. 2). In der Gemeinde Castiel (ERDIN 1984) und im Schächental (AMACHER 1983) konnte auf solchen Flächen eine Zunahme der Blaiken-Bildung festgestellt werden. Die Thematik wurde auch im Bündner Großen Rat (BÜNDNER ZEITUNG 1982) politisch aufgegriffen. Aus Fig. 2/3 kann weiter ersehen werden, daß mit einer Erhöhung der Brandgefahr vor allem in Gebieten mit einer fortgeschrittenen Brachlandentwicklung (Vernamiège, Chandolin, Palagnedra) zu rechnen ist, weil dort bereits in Siedlungsnähe großflächig gras-/krautschicht-dominante Formationen auftreten und diese Gebiete von ihrer naturräumlichen Disposition (Trockenheit) brandgefährdet sind. In unseren Untersuchungen konnten wir feststellen, daß Brachland von der Ortsbevölkerung einheitlich im Zusammenhang mit der Brandgefahr problematisiert wird.

\subsection{Brachland als Veränderung der Wirtschaftsflächen}

Viele der bisherigen Beurteilungen und Problemformulierungen (z. B. SURBER et al. 1973) beschäftigen sich mit dieser Umwelt-Dimension. Primär heißt Brachlegung nichts anderes, als da $B$ die Landwirtschaft Flächen aufgibt, an deren Bewirtschaftung sie (oder mindestens die ortsansäßigen Bauern) kein Interesse mehr hat. Für die Forstwirtschaft heißt jedoch Brachland ein Waldflächenzuwachs, der nicht in allen Fällen erwünscht ist und der wegen des Forstgesetzes im jetzigen Zeitpunkt nicht rückgängig gemacht werden kann. Diese beiden Positionen gilt es genauer zu überprüfen.

Aus landwirtschaftlicher Sicht ist die Unterscheidung in reversibles und irreversibles Brachland (mit Holzgewächsen) sowie der die Bewertung nach ihrer Bodeneignung wichtig. Fig. 3 zeigt, daß nur ein beschränkter Anteil der 1950 noch genutzten Flächen bereits irreversibel verwaldet ist. Im ersten Stadium der Brachlandentwicklung werden zudem die schlechten Böden aufgelassen (Fig. 2), so daß eine Problematisierung aus landwirtschaftlicher Sicht nicht nötig erscheint (z. B. Riemenstalden, Camuns, Castiel). Wenn jedoch größere Flächen guten landwirtschaftlichen Bodens aufgelassen werden, sind erste kritische Bemerkungen vertretbar. In den Gemeinden Ramosch und Schlans sind solche Entwicklungen zu beobachten, wobei erst ein kleiner Anteil irreversibel verholzt ist. Bedeutender ist der Kulturlandverlust in Gemeinden der Kantone Wallis und Tessin. Hier erscheinen planerische Eingriffe mit dem Ziel einer Minimalbewirtschaftung erforderlich.

Aus forstlicher Sicht vertritt BOSSHARD (in SURBER et al. 1973) die Ansicht, daß der durch Brachlegung verursachte Waldflächenzuwachs für die Forstwirtschaft uninteressant sei, da es sich meist um sogenannte Grenzertragsböden handle. Generell stimmen wir dieser Ansicht zu, doch möchten wir dem zwei Überlegungen gegenüberstellen: Erstens setzt die Brachlandentwicklung meist in der subalpinen Stufe (Maiensäße) ein, wo großflächig forstwirtschaftlich 
interessante, oftmals sogar erschlossene NadelholzFormationen aufwachsen, und zweitens ist in Gemeinden mit einer fortgeschrittenen Brachlandentwicklung auch auf guten Böden ein beträchtlicher Waldzuwachs festzustellen (Fig. 3), der zwar in seiner Artenzusammensetzung zumindest in den unteren Lagen für eine Nutzung ungeeignet ist. Leider ist in diesen Problemgebieten (z. B. Chandolin, Vernamiège, Palagnedra) die Forstwirtschaft personell und infrastrukturell für solche Mehraufgaben wenig ausgerüstet. Wichtig scheint uns für die Forstwirtschaft auch die Tatsache, daß der auf Brachland aufwachsende Wald überwiegend Privatwald ist.

Auf produktiven, maschinell bewirtschaftbaren Böden ist bei weiterem Fortschreiten der Brachlandentwicklung mit Konflikten zwischen Land- und Forstwirtschaft zu rechnen. Charakteristisch ist, daß diese Konflikte zuerst in der Maisensäßstufe auftreten (z. B. Schlans). In allen untersuchten Gebieten, die Ausnahme bildet vielleicht Vernamiège (Fig. 3), scheinen diese Konflikte im jetzigen Zeitpunkt noch lösbar. Da der Handlungsspielraum für planerische Eingriffe jedoch durch den natürlichen Ablauf der Vegetationssukzession begrenzt ist, sollten gerade heute in Problemgebieten Waldflächenentwicklungspläne (vgl. Beitrag von PFISTER) im Rahmen der Richt- und Nutzungsplanung erstellt werden. Der Handlungsspielraum erscheint uns in den Kantonen Tessin und Graubünden beschränkter als etwa im trockenen Kanton Wallis.

\subsection{Brachland als Erlebnisbereich}

Gerade von geographischer Seite, die sich auch intensiv mit wahrnehmungstheoretischen und umweltpsychologischen Fragen auseinandersetzt, sind abschließend einige Beurteilungen zu diesem Thema erwünscht. Erste Ansätze liefern uns die Arbeiten von HARD (1975) und NOHL (1976), die in Deutschland die Wirkung des Brachlandes auf Nahraum- und Fernsichterlebnis untersuchten, wobei die gewonnenen Resultate nicht bedingungslos auf schweizerische Verhältnisse übertragen werden können. Wir möchten voranstellen, daß die folgenden Überlegungen nur aus den Wertvorstellungen einer Industriegesellschaft begründbar sind und deshalb in land- und forstwirtschaftlich orientierten Kreisen des Berggebietes nicht unbedingt auf Verständnis stoßen, was übrigens auch einzelne Diskussionsbeiträge an diesem Symposium zeigten. Aus realistischer Sicht muß jedoch heute die Verflechtung Berggebiet - städtische Industriegesellschaft als Tatsache hingenommen werden.

Das Landschaftsbild ist einerseits eine wichtige Ressource für den alpinen Sommertourismus, was in vielen Werbeprospekten der Verkehrsvereine zum Ausdruck kommt. Diese Werbung mit einer intakten, traditionsverwurzelten Agrarlandschaft läßt sich bei einer kleinflächigen, parzellenartigen Brachlandvertei- lung noch gut vertreten. Diskrepanzen zwischen Anspruch und Wirklichkeit treten jedoch auf, wo die Brachlandentwicklung bereits so weit fortgeschritten ist, da $B$ in Dorfnähe flurartig Brachland auftritt (z. B. Vernamiège, Chandolin). Der «Zerfall der Kulturlandschaft) wird schon seit BERNHARD (1928) mit einem «Kulturabbau» gleichgesetzt, was aus begrifflicher Sicht verständlich erscheint. Das Dilemma ist nun, $\mathrm{da} ß$ Praktiker, die mit den örtlichen Problemen vertraut sind, diesen Traditionsabbau längst überwunden haben, der Tourist aber mit seinen spezifischen Idealen den gleichen Schritt nicht unbedingt vollziehen kann. Vor allem im Kanton Wallis sollte die Brachlandentwicklung einmal unter diesem Aspekt betrachtet werden.

Besonders wichtig erscheint uns der Aspekt «Brachland als Freiraum für die Gesellschaft». Durch die Arbeitszeitverkürzung in der Wirtschaft sowie durch neuere gesellschaftliche Entwicklungen (Ferienhaustourismus, Alternativbewegung usw.) entsteht ein Bedürfnis nach Freiräumen, dem vielleicht gewisse Brachlandflächen gerecht werden könnten. Im Kanton Tessin sind diese Gedanken schon längst keine Theorie mehr: Die lichtungsartige Brachlandverteilung in der Montistufe (kleine, überblickbare Raumeinheiten) schafft hier besonders günstige Voraussetzungen, wobei die wichtigste Bedingung das Vorhandensein von kaufbaren Gebäuden ist. Ein ideal-typisches Beispiel beschreibt MAHLER (1981) im Centovalli. Die Stiftung TERRA VECCHIA, die sich mit der Therapie und Integration von Drogenabhängigen beschäftigt, fand in einer aufgelassenen Siedlung die idealen Verhältnisse für den Aufbau eines Zentrums. Zentrale Begriffe waren etwa Romantik, Abgeschiedenheit, Pioniergeist und Naturnähe. Den durch diese «Freiraum-Nutzung» auftretendenden Konflikten, die keinesfalls unterschätzt werden dürfen, sollte mit einer aktiven Planung begegnet werden.

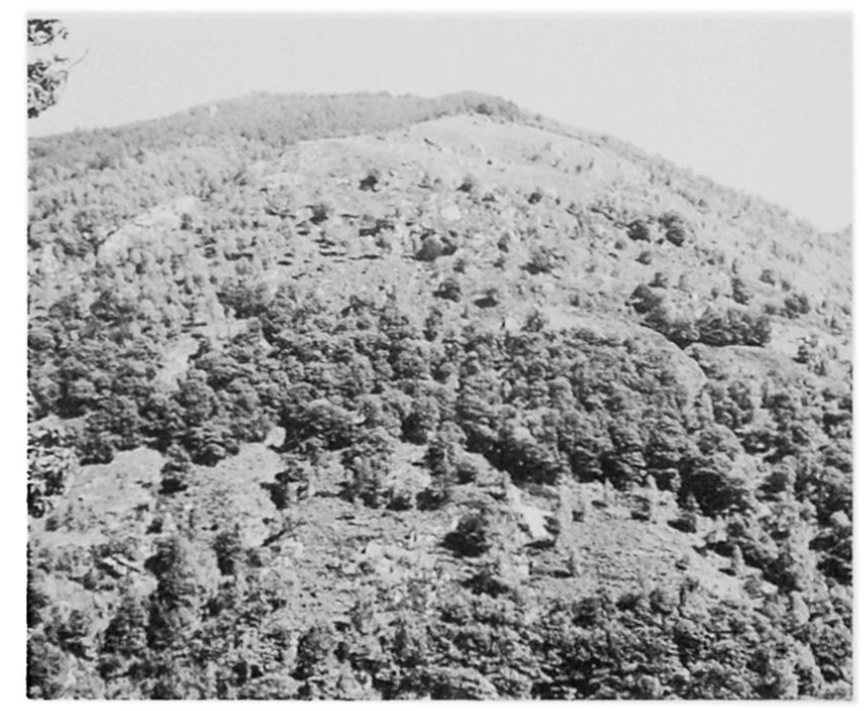

Brachland als Raum unkontrollierter Waldflächenentwicklung (Val Onsernone, Aufnahme P. Walther). 


\section{Zusammenfassung, Summary}

Basierend auf den Resultaten eines Projektes und anderen, für einmal bewußt geographischen Arbeiten, wird zur Einführung in die Thematik dieses Symposiums eine integrierte Theorie zur Beurteilung der Brachlandentwicklung 1950-1980 vorgeschlagen. Dargestellt wird einerseits eine Theorie der Brachlandentwicklung (Kap.2), die vor allem auf nationaler und kantonaler Ebene zur Ausscheidung aktueller und künftiger Problemgebiete dient. Andererseits wird «Brachland als Umweltveränderung» von der begrifflichen Seite durchleuchtet und ein Vorschlag zur Bildung planerisch verwendbarer «Brachland-UmweltKategorien" unterbreitet (Kap.3). Die Kombination der beiden Ansätze erlaubt abschließend erste Versuche einer Beurteilung und Bewertung der Umweltveränderungen und der daraus erwachsenden Konflikte, die im Rahmen der Richt- und Nutzungsplanung gelöst werden sollten (Kap.4). Eine stärker auf die Praxis ausgerichtete Darstellung dieser Ansätze haben wir in einer zusätzlichen Arbeit veröffentlicht (HUGENTOBLER/WALTHER 1983).
Based on the results of an extensive project on land abandonment in various parts of the Swiss Alps and other geographical publications, an integrated theory is proposed for the evaluation of abandoned land in mountain areas as introduction to the Symposium. The features under study serve as a theory of land-use changes on the one hand, which allows to define actual and future problem regions on a national and regional level (chapter 2). On the other hand, abandoned land is understood as an element of our environment and a concept is proposed for practicable «abandoned land - environment categories» for planning purposes (chapter 3 ). The combination of the two aspects leads to preliminary conclusions and evaluations regarding the environmental changes and the resulting conflicts which have to be solved by the official planning agencies under the new areal planning act (chapter 4 ). The aspects dealing primarily with the applications of these findings to planning and managing purposes are published in an additional article (HUGENTOBLER/WALTHER, 1983).

\section{Literaturverzeichnis}

AMACHER, E. (1983): Die Nutzungsänderung auf Wildheuflächen im Schächental und ihre ökologischen Auswirkungen. Dipl. Arb. Geogr. Inst., Zürich.

BARDELEBEN, R. v., GEKLE, L. (1978): Nutzen- und Schadenkomponenten bei gepflegter und ungepflegter Brache unter Berücksichtigung verschiedener Flächenumfänge, Standorte und Vorrangfunktionen, Münster.

BERNHARD, H. (1928): Die Wirtschaftsprobleme des Valle Maggia (Tessin) als typischen Gebirgsentvölkerungsgebietes. In: Schriften d. Schw. Ver. f. Innenk. u. industr. Landw., Nr. 36.

BÜNDNER ZEITUNG (1982): Kommentar zu einer Sitzung des Bündner Großen Rates, 3.6.82, Chur.

DARBELLAY, CH. (1980): Agriculture et société: un nouveau contrat, Charrat.

ERDIN, CH.: Prozesse und Dynamik der Erosion auf brachgelegten Bergwiesen im Kt. GR (Arbeitstitel). Dipl. Arb. Geogr. Inst., Zürich (in Vorbereitung).

EVD (1976): 5. Landwirtschaftsbericht, Bern.

HARD, G. (1975): Brache als Umwelt. In: Lands. u. Stadt, Nr. 4, S. $145-153$.

HIRZEL, W. (1983): Brachlandentwicklung im Val d'Hérens, Dipl. Arb. Geogr. Inst., Zürich.

HUGENTOBLER, F., WALTHER, P. (1983): Die Erfassung von Brachlandsituationen in der Richt- und Nutzungsplanung. In: Physisch-geogr. Serie Nr. 11, Geogr. Inst., Zürich.

LANDTWING, K. (1979): Die Arbeiterbauern des Lonzawerkes Visp und ihre Kulturlandschaft. Diss. Universität Zürich.
MAHLER, W. (1981): Brachland in den Centovalli. Dipl. Arb. Geogr. Inst., Zürich.

MAHRER, F. (1976): Abgrenzungsnormen zur Erfassung der Waldfläche im Schweizerischen Landesforstinventar. In: Berichte EAFV, Nr. 167, Birmensdorf.

NOHL, W., SCHARPF, H. (1976): Erlebniswirksamkeit von Brachflächen. In: KTBL: Brachflächen in der Landschaft, Münster-Hiltrup.

RÄBER, P. (1981): Berglandwirtschaft und Brachland im Lugnez. Dipl. Arb. Geogr. Inst., Zürich.

RUF, M. (1982): Ursachen und Auswirkungen der Brachlegung von Kulturland im Schanfigg. Dipl. Arb. Geogr. Inst., Zürich.

SCHORI, U. (1982): Landwirtschaft und Brachland im Unterengadin. Dipl. Arb. Geogr. Inst., Zürich.

SURBER, E., ET AL. (1973): Das Brachlandproblem in der Schweiz. Berichte EAFV, Nr. 112, Birmensdorf.

WALTHER, P., GRESCH, P., HAEFNER, H. (1982): Die Brachlandentwicklung im Obergoms 1970-1980. In: Geogr. Helv. Nr. 4. S. 3-14.

WALTHER, P., JULEN, S. (1983): Brachlandsituationen und Waldflächenentwicklung im Schweizer Alpenraum (Arbeitstitel). Erscheint in Berichte EAFV, Birmensdorf.

WEICHHART, H. (1980): Auf dem Weg zu einer Theorie der Gesellschaft-Umwelt-Beziehung. In: Österr. geogr. Mitt., Bd. 122, S. 49-69, Wien. 